\title{
Buckling resistance evaluation of steel beam-columns using refined General Method approach
}

\author{
Zbigniew Stachura ${ }^{1, *}$ and Marian A. Gizejowski ${ }^{1}$ \\ ${ }^{1}$ Warsaw University of Technology, Faculty of Civil Engineering, Division of Metal Structures, Warsaw, Poland
}

\begin{abstract}
Different aspects of Eurocode 3 General Method (GM) approaches are discussed in this paper. The purpose of present study is to improve the application of GM approach for both beam-columns without intermediate lateral-torsional restraints and with these restraints. The results from the proposed GM are compared with those from Eurocode 3-1-1 interaction equations according to Method 1 and Method 2. A better consistency between the developed GM approach and the Eurocode's interaction equation approach than Eurocode $3 \mathrm{GM}$ approach is observed.
\end{abstract}

\section{Introduction}

Eurocode's design rules related to the buckling resistance of beam-columns are constructed in two ways. Firstly, the interaction interpolation equations are presented in which two independent buckling reduction factors are used, and based on the slenderness ratios defined independently for compression and bending about $y-y$ axis. This approach is verified with use of finite element simulations and validated using available experimental data [1,2]. Secondly, the so-called General Method (GM) is presented as an alternative to the former one [3]. The slenderness in the latter approach is generalised taking the form $\bar{\lambda}_{o p}=\sqrt{\alpha_{u l t, i p} / \alpha_{c r, o p}}$. It tends to account for an integrated effect of flexuraltorsional (FT) buckling under combined compression and bending. Part 1-6 of Eurocode 3 [4] specifies the socalled EC-1-6 GM (MNA/LBA EC3-1-6 approach) while Part 1-1 of Eurocode 3 [5] recommends EC3-1-1 GM (GMNIA/LBA EC-1-1 approach). The difference between them is as follows:

1. MNA/LBA EC3-1-6 recommends such a GM in which the parameters of Materially Nonlinear Analysis (MNA) are used for the calculation of the in-plane inelastic design load multiplier $\alpha_{\text {ult, } i p}$ (without the inplane geometrically nonlinear effects taken into account) and Linear Buckling Analysis (LBA) are used for the calculation of the nominal elastic critical load multiplier $\alpha_{c r}$ taking all elastic instability modes into account.

2. GMNIA/LBA EC3-1-1 recommends such a GM in which the parameters of in-plane Geometrically and Materially Nonlinear Analysis of Imperfect structure (GMNIA) are used for the calculation of the in-plane inelastic design load multiplier $\alpha_{u l t, i p}$ and Linear Buckling Analysis (LBA) are used for the out-of-plane nominal elastic critical load multiplier $\alpha_{c r, o p}$.
Different aspects of Eurocode $3 \mathrm{GM}$ approaches are discussed and followed by the author's proposal of GM in which a better consistency between the developed GM approach and the Eurocode 3 interaction equation approach is observed. This paper is a continuation of previous authors' studies [6-9] on refining the GM approach for both beam-columns without intermediate lateral-torsional restraints and with these restraints.

Recent international research with the use of various proposals is also related to the assessment of the beamcolumn resistance. The first of them is based on a direct application of the Ayrton-Perry approach [10-12] where a generalised slenderness concept is applied. The second proposal [13] is the so-called Overall Imperfection Method and it is adequate for computer-aided design methodology which is based on advanced elastic beamcolumn finite element method. Third example includes the Overall Interaction Concept and is based on the interaction of local and global instability resistance and the definition of a generalised relative slenderness [14]. Other example is the Direct Strength Method which is dedicated for cold-formed steel member [15]. Comparison of the General Method with the Overall Method for the out-of-plane stability of members with lateral restraints can be found in [16].

\section{Discussion of Eurocode 3 GM approaches}

Assessment of the beam-column elastic critical state may be based on the conventional Linear Eigenvalue Analysis (LEA) in which the effect of initial stresses from Linear Analysis (LA) is accounted for or it is based on the Nonlinear Eigenvalue Analysis (NEA) in which the effects of axial force on the in-plane displacements and the in-plane displacements on the out-of-plane buckling load are considered [17].

* Corresponding author: z.stachura@il.pw.edu.pl 
The MNA/LBA EC3-1-6 approach requires in general to use of NEA in order not to miss the effect of in-plane buckling. Figure 1 shows the difference between two possible situations may take place, namely when a) $\chi_{y}>\min \left(\chi_{z}, \chi_{T}\right)$ or b) $\chi_{y}<\min \left(\chi_{z}, \chi_{T}\right)$. The former situation is typical for beam-columns without lateraltorsional (LT) restraints while the latter - for beamcolumns with LT restraints.

Only when using NEA it gives the MNA/LBA EC31-6 approach results coinciding with those obtained from the interaction buckling resistance utilization factor approach of clause 6.3.3 [5], namely the buckling resistance in compression is equal to $N_{b, R k}=\chi N_{c, R k}$ (in absence of bending moments $M_{y, E d}$ and $M_{z, E d}$ ). In case of zero bending moments, the buckling resistance reduction factor becomes $\chi=\min \left(\chi_{y}, \chi_{z}, \chi_{T}\right)$. The difference may appear in the buckling bending resistance equal to $M_{b y, R k}=\chi_{L T} M_{c y, R k}$ (in the absence of axial force $N_{E d}$ and bending moment $M_{z, E d}$ ). It yields from the fact that the slenderness ratio $\bar{\lambda}_{L T}=\sqrt{M_{c, R k} / M_{c r}}$ is calculated using the critical moment $M_{c r}$ that may be of a different value calculated from LEA and NEA. Such a difference is especially visible for wide flange I-sections of in-span unrestrained members for which the effect of in-plane displacements is considered in NEA, uplifting the value of $M_{c r}$ in comparison to that obtained from LEA. On the other hand, when wide flange I-section beam-columns with LT restraints are considered, the critical moment from NEA becomes of a substantially larger value than that from LEA, so that the relative slenderness ratio may become less than 0,2 , so that the buckling reduction factor $\chi_{o p}=\chi_{L T}=1$ and the buckling resistance $M_{b y, R k}=$ $=\chi_{o p} M_{c y, R k}=M_{c y, R k}$. The conclusion is that for the MNA/LBA EC3-1-6 approach it is more rational to use the elastic critical load factor obtained from NEA. Despite the buckling resistance being evaluated more correctly in extreme cases of bending under $M_{y, E d}$ and compression $N_{E d}$, the resistance for all the intermediate cases of $\left(M_{y, E d}, N_{E d}\right)$ remains evaluated on an unsafe side.

Contrarily, the GMNIA/LBA EC3-1-1 approach according to clause 6.3.4 [5] takes into account the inplane second order effects and the influence of imperfections on the buckling resistance for the calculation of $\alpha_{u l t, i p}$ and LEA for the calculation of $\alpha_{c r, o p}$. Although such an approach is more realistic, it gives inconsistency if compared to that of the interaction buckling resistance utilization factor approach of clause 6.3.3 [5]. The GMNIA/LBA EC3-1-1 approach gives a quite conservative estimate of the buckling resistance in case of axial compression or when the axial force is accompanied by the bending moment, i.e. giving lower values than those obtained from the interaction buckling resistance utilization factor approach. Let us consider the axial compression case of HEB 300 section of the steel grade $\mathrm{S} 235$ with the dimensionless stress resultants $n_{E d, 0}=0,10$ [see Eq. (12)] and the in-span unrestrained beam-column with the slenderness ratios $\bar{\lambda}_{i p}=\bar{\lambda}_{y}=1,167$ and $\bar{\lambda}_{o p}=\max \left(\bar{\lambda}_{z}, \bar{\lambda}_{T}\right)=2,0$. The in-plane dimensionless resistance from GMNIA is equal to the redution factor $\chi_{y}=0,497$ and $\alpha_{u l t, i p}=\chi_{y} / n_{E d, 0}=4,97$. On the other hand, the out-ofplane critical load multiplier is equal to $\alpha_{c r, o p}=$ $=1 /\left(n_{E d, 0} \bar{\lambda}_{o p}^{2}\right)=2,50$ and the out-of-plane slenderness ratio $\bar{\lambda}_{o p}=\sqrt{\alpha_{u l t, i p} / \alpha_{c r, o p}}=1,41$. As a results the dimensionless buckling reduction factor is equal to $\chi_{o p}=0,346$ and finally $\alpha_{u l t, o p}=\chi_{o p} \alpha_{u l t, i p}=1,72$ being of a substantially lower value than that from the Eurocode's interaction equation approach [5] where $\chi_{z} / n_{E d, 0}=1,96$ is calculated.
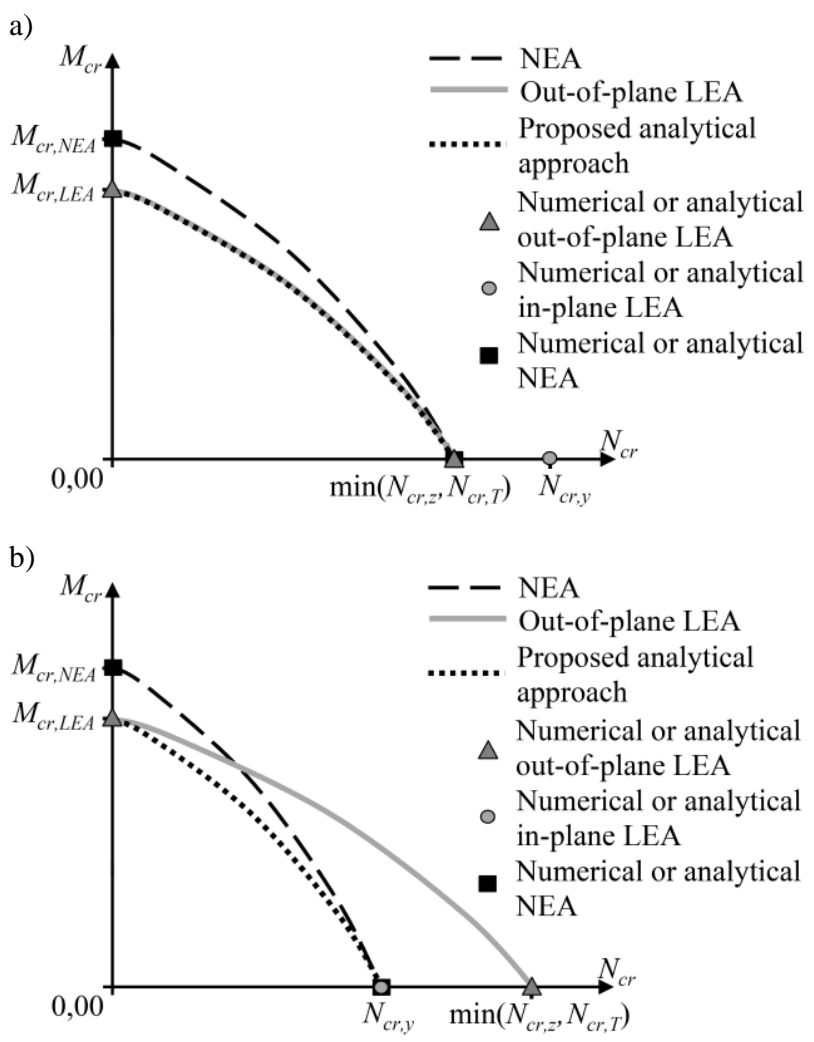

Fig. 1. Two possible critical state curves obtained from LEA and NEA, a) critical force related to $\min \left(\chi_{z}, \chi_{T}\right)$ governs, b) critical force $\chi_{y}$ governs.

It is important to note that in this type of approach the effect of imperfections is considered twice in their maximum magnitude, firstly the maximum effect of in-plane imperfections is considered in the calculation of $\alpha_{u l t, i p}$ and secondly, the maximum effect of out-of-plane imperfections is considered in the calculation of $\chi_{o p}$. In order to be in line with the general recommendation of Eurocode 3 that the effect of imperfections should be considered in one buckling direction only, the proposal presented by the authors in [6] is based on the calculation of $\alpha_{u l t, i p}$ from GMNA instead of GMNIA, while the effect of imperfections is considered through the imperfection factor interpolated between those related to the LT buckling curve and to the FT buckling curve. In the example considered above, it gives for compression $\quad \alpha_{u l t, i p}=1 /\left(n_{E d, 0} \bar{\lambda}_{i p}^{2}\right)=1 /\left(n_{E d, 0} \bar{\lambda}_{y}^{2}\right)=7,34$ and $\bar{\lambda}_{o p}=\sqrt{\alpha_{u l t, i p} / \alpha_{c r, o p}}=1,71$ and $\chi_{o p}=0,254$. Finally 
dimensionless buckling resistance is equal to $\alpha_{u l t, o p}=\chi_{o p} \alpha_{u l t, i p}=1,86$ and is much closer to that of 1,96 obtained from the Eurocode's interaction equation approach [5].

The purpose of present study is to further improve the application of GM approach for both beam-columns without intermediate LT restraints and with these restraints.

\section{Basis of proposed GM approach for the beam-column buckling resistance}

Let us consider the beam-column element $i-k$ isolated from the structural systems with end moments obtained from GNA and in-span loads consisting of concentrated $Q_{j d}$ forces and distributed loads $q_{j d}(x)$ with the left ordinate $q_{j i, d}$ and right coordinate $q_{j k, d}$, as presented in Figure 2a. The element shown in figure is subjected to only one concentrated force component at the distance $x_{Q j}$ while the coordinates of distributed in-span load component are $x_{q, j i}$ and $x_{q, j k}$, for the load ordinates $q_{j i, d}$ and $q_{j k, d}$, respectively. The values of in-span loads correspond to the end moments so that it is a reference force state identified by the subscript " 0 ". The end moments and in-span loads corresponding to the in-plane failure mode are defined through a load factor $\alpha_{u l t, i p, k}$ where the subscript " $k$ " defines here the characteristic values:

$$
\begin{gathered}
M_{y i, u l t}=M_{y, h, u l t, E k}=\alpha_{u l t, i p, k} M_{y i, E d}=\alpha_{u l t, i p, k} M_{y, h, E d} \\
M_{y k, u l t}=\alpha_{u l t, i p, k} M_{y k, E d}=\alpha_{u l t, i p, k} \psi_{y} M_{y, h, E d} \\
Q_{j, u l t}=\alpha_{u l t, i p, k} Q_{j d} \\
q_{j i, u l t}=\alpha_{u l t, i p, k} q_{j i, d}, \quad q_{j k, u l t}=\alpha_{u l t, i p, k} q_{j k, d}
\end{gathered}
$$

The $x$-coordinate is measured from the left-hand side support of the beam-column element. The general loading case can be treated as a superposition of two elementary cases. Let us assume at the beginning that the end moments produce a linear moment gradient along the element length through the superposition of $x$-dependent "hogging moment" components, namely "symmetric hogging" $M_{y, h s, E d}$ and "antisymmetric hogging" $M_{y, h a, E d}$. The maximum displacement in the $z$-axis direction is calculated from the superposition of displacement components corresponding to $M_{y, h s, E d \text {, say }}$ $\delta_{z, h s}$ in the half span length and to $M_{y, h a, E d}$, say $\delta_{z, h a}$ in the quarter span length. The span loads produce the $x$-dependent first order bending moment components of "symmetric sagging" $M_{y, s s, E d}$ and "antisymmetric sagging" $M_{y, s a, E d}$ as well as corresponding displacement components, in the half span length $\delta_{z, s s}$ and in the quarter span length $\delta_{z, s a}$, respectively. Finally, the approximation of nonlinear effects on the moment gradient is accounted for by the application of amplification factors to the said above displacement components and the adoption of representative shape functions for modelling the member symmetric and antisymmetric deflected profiles in order to find the $P-\delta_{z}$ effect related moments. The stress resultant, in-span loads and displacement load effects corresponding to both symmetric and antisymmetric components due to the identified above hogging and sagging cases are shown in Figure 2.

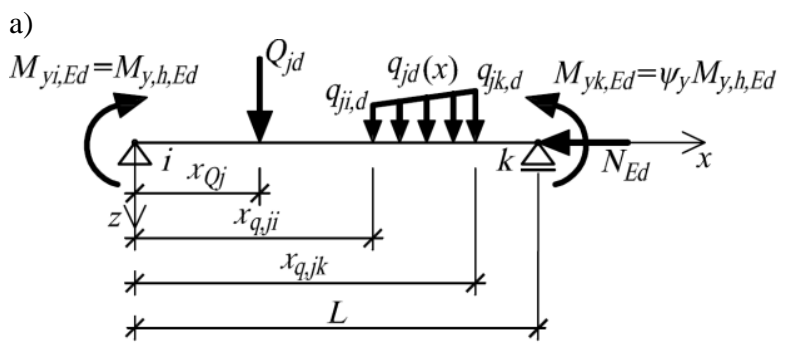

b)
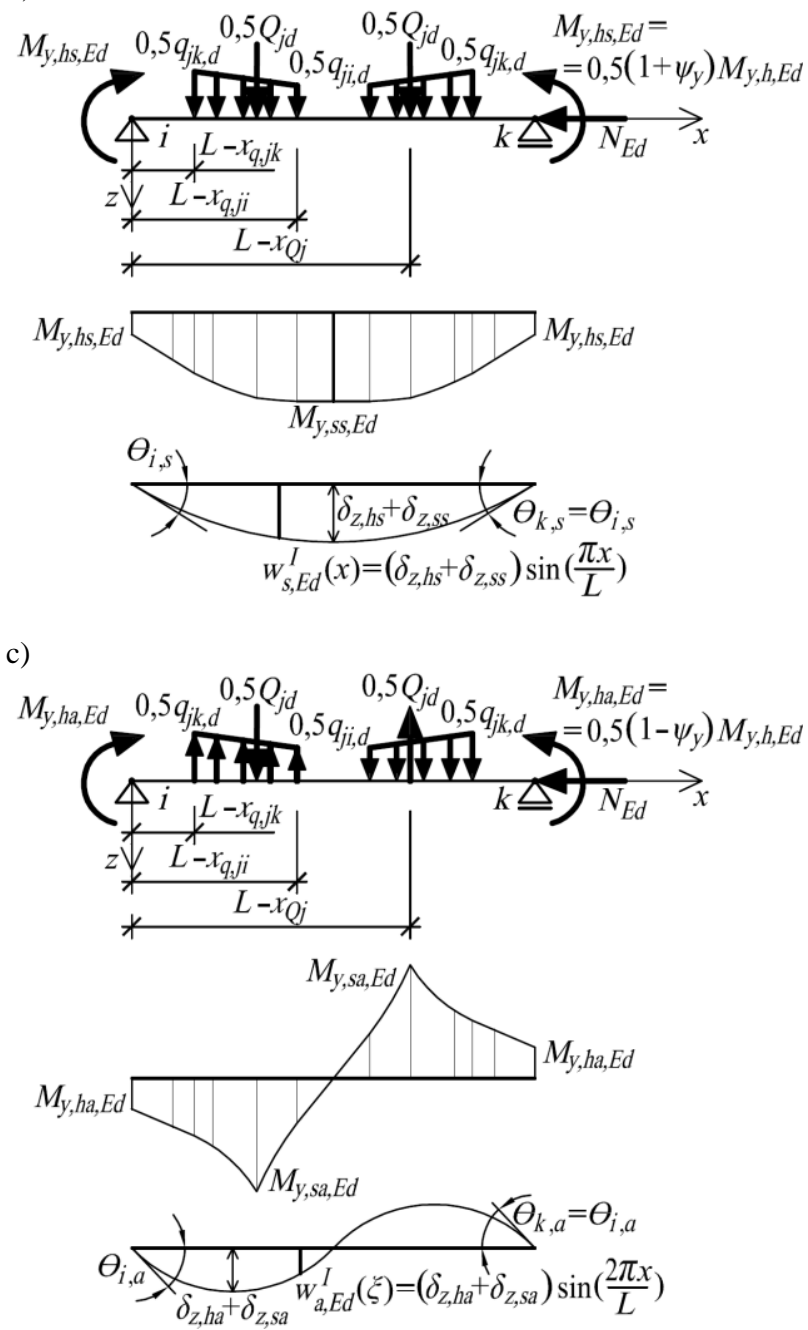

Fig.2. End moments and in-span load as well as displacement components, a) general, b) symmetric components, c) antisymmetric components.

\subsection{In-plane resistance}

The resistance utilization factor referred to the reduced section moment resistance $M_{y N, R k}$ in the ultimate limit state of class 1 and 2 cross section members may be written down using the format given below: 


$$
U_{M y}+U_{P-\delta_{z}}=\left\{\begin{array}{cl}
1 & \text { for } 0 \leq \alpha_{n} \leq 0,5 a \\
\frac{1-\alpha_{u l t, k} n_{E d, 0}}{1-0,5 a} & \text { for } 0,5 a<\alpha_{n}
\end{array}\right.
$$

In the following, the load case without the in-span load components is considered in details in which there are the following linear and nonlinear utilization factor components expressed for the GMNA failure criterion:

$$
\begin{array}{r}
U_{M y}=0,5 \alpha_{u l t, k} m_{y, E d, 0}\left[1+\psi_{y}+\left(1-\psi_{y}\right)\left(1-\xi_{\max }\right)\right] \\
U_{P-\delta_{z}}=\alpha_{u l t, k} n_{E d, 0}\left[\beta_{\delta, s h} \eta_{y, s h, E d} \bar{w}_{s}\left(\xi_{\max }\right)+\right. \\
\left.+\beta_{\delta, a h} \eta_{y, a h, E d} \bar{w}_{a}\left(\xi_{\max }\right)\right]
\end{array}
$$

In the above equations, the load multiplier $\alpha_{u l t, k}=\alpha_{u l t, i p, k}$ is the factor calculated for a given stress resultants ratio $\alpha_{n}$ (or $\alpha_{m}$ ) defined as follows:

$$
\alpha_{n}=\frac{n_{E d, 0}}{m_{y, E d, 0}}, \quad \alpha_{m}=\frac{m_{y, E d, 0}}{n_{E d, 0}}=\frac{1}{\alpha_{n}}
$$

The multiplier $\alpha_{u l t, k}=\alpha_{u l t, i p, k}$ allows for the evaluation of the in-plane ultimate limit state identified by the load effects $\left(n_{E d, u l t} ; m_{y, E d, u l t}\right)$. The in-plane resistance curve expressed in the dimensionless coordinates is constituted by points with two following coordinates $\left(n_{E d, u l t}=\alpha_{u l t, k} n_{E d, 0} ; m_{y, E d, u l t}=\alpha_{u l t, k} m_{y, E d, 0}\right)$.

Furthermore, the $a$-parameter in Eq. (5) is the I- and $\mathrm{H}$-sections shape factor given in [5] and there is introduced the dimensionless member axis coordinate $\xi=2 x / L$ (where $L$ is a length of the element), in particular the coordinate $\xi=\xi_{\max }$ of the most stressed section for which Eq. (5) is to be satisfied. The dimensionless maximum displacement components, symmetric and antisymmetric are for the end moments case expressed as follows:

$$
\eta_{y, s h, E d}=\frac{N_{p l, R k} \delta_{z, s h}}{M_{p l, y, R k}}, \quad \eta_{y, a h, E d}=\frac{N_{p l, R k} \delta_{z, a h}}{M_{p l, y, R k}}
$$

where $N_{p l, R k}$ - characteristic value of plastic resistance to compression,

$M_{p l, y, R k}$ - characteristic value of plastic resistance to bending moments about $y-y$ axis.

In the GM approach proposed hereafter, the amplification factors are restricted to certain levels of the member relative in-plane slenderness ratio and given as below:

$$
\beta_{\delta, s h}= \begin{cases}\frac{1}{1-\bar{\lambda}_{y}^{2} \alpha_{u l t, k} n_{E d, 0}} & \text { for } \bar{\lambda}_{y} \leq 1 \\ \frac{1}{1-\alpha_{u l t, k} n_{E d, 0}} & \text { for } \bar{\lambda}_{y}>1\end{cases}
$$

$$
\beta_{\delta, a h}=\left\{\begin{array}{cc}
\frac{1}{1-0,25 \bar{\lambda}_{y}^{2} \alpha_{u l t, k} n_{E d, 0}} & \text { for } \bar{\lambda}_{y} \leq 2 \\
\frac{1}{1-\alpha_{u l t, k} n_{E d, 0}} & \text { for } \bar{\lambda}_{y}>2
\end{array}\right.
$$

and the dimensionless maximum stress resultants are written down as follows:

$$
n_{E d, 0}=\frac{N_{E d}}{N_{p l, R k}}, \quad m_{y, E d, 0}=\frac{M_{y, h, E d}}{M_{p l, y, R k}}
$$

The shape functions are adopted herein in the form of two lowest buckling modes, symmetric and antisymmetric ones and given by the following wellknown expressions:

$$
\bar{w}_{s}=\sin (0,5 \pi \xi), \quad \bar{w}_{a}=\sin (\pi \xi)
$$

Using the adopted assumptions, the consistency in the calculation of the compression buckling resistance is obtained between the Eurocode's interaction equations [5] and the proposed GM approach.

\subsection{Elastic out-of-plane buckling}

A similar form of the interaction equation as in the case of a single element under uniform bending with an improvement to the critical load factor evaluation for beam-columns with LT restraints is adopted:

$$
\begin{aligned}
& \left(\frac{\alpha_{c r} M_{y, E d, h}}{M_{c r, N}}\right)^{2}=\left(1-\frac{\alpha_{c r} N_{E d}}{N_{b i f, y}}\right)\left(1-\frac{\alpha_{c r} N_{E d}}{N_{b i f, z}}\right)\left(1-\frac{\alpha_{c r} N_{E d}}{N_{b i f, T}}\right) \\
& \frac{M_{c r}}{M_{c r, N}}=\frac{\left(\frac{1+\psi_{y}}{2}\right)}{\left(\frac{1+\psi_{y}}{2}\right)+\left(\frac{1-\psi_{y}}{2}\right)^{3}\left(\frac{\bar{\lambda}_{L T, a}}{\bar{\lambda}_{L T, s}}\right)^{2}}+ \\
& +\frac{\left(\frac{1-\psi_{y}}{2}\right)^{3}\left[\left(\frac{\bar{\lambda}_{L T, a}}{\bar{\lambda}_{L T, s}}\right)^{2}-\left(0,23 \frac{\bar{\lambda}_{z}}{\bar{\lambda}_{z, 0}}\right)^{2} \alpha_{E d, 0} \bar{\lambda}_{z}^{2}\right]}{\left(\frac{1+\psi_{y}}{2}\right)+\left(\frac{1-\psi_{y}}{2}\right)^{3}\left(\frac{\bar{\lambda}_{L T, a}}{\bar{\lambda}_{L T, s}}\right)^{2}}
\end{aligned}
$$

where the values $M_{c r}$ and $N_{b i f, i}$ are calculated numerically according to LEA, using the free of charge software LTBeamN for the purpose of this study (except $N_{b i f, y}$ that is calculated analytically) and $\bar{\lambda}_{L T, s}, \bar{\lambda}_{L T, a}-\mathrm{LT}$ buckling slenderness ratios for $\psi_{y}=1$ and $\psi_{y}=-1$, respectively; $\bar{\lambda}_{z, 0}-$ the flexural slenderness ratio about $z-z$ axis corresponding to the unrestrained case.

It is important to note that for LT unrestrained beamcolumns Eq. (15) becomes of that given in [18]. 


\subsection{The beam-column buckling resistance}

The characteristic buckling resistance for proposed GM approach is calculated with the use of the following equation [6]:

$$
\alpha_{b, u l t, k}=\chi_{F T, k} \alpha_{u l t, k}
$$

The characteristic value of the reduction factor $\chi_{F T, k}$ is defined in the well-known Ayrton-Perry format:

$$
\begin{gathered}
\chi_{F T, k}=\left(\phi_{F T}+\sqrt{\phi_{F T}^{2}-\bar{\lambda}_{F T}^{2}}\right)^{-1} \\
\phi_{F T}=0,5\left(1+\alpha_{F T}\left(\bar{\lambda}_{F T}-0,2\right)+\bar{\lambda}_{F T}^{2}\right)
\end{gathered}
$$

in which the relative slenderness ratio $\bar{\lambda}_{F T}$ and the imperfection factor $\alpha_{F T}$ are calculated from the formulas [6]:

$$
\begin{gathered}
\bar{\lambda}_{F T}=\sqrt{\frac{\alpha_{u l t, k}}{\alpha_{c r}}} \\
\alpha_{F T}=\frac{\alpha_{L T}}{1+\alpha_{n}}+\frac{\alpha_{i}}{1+\alpha_{m}}
\end{gathered}
$$

where the multipliers $\alpha_{u l t, k}$ and $\alpha_{c r}$ are calculated from the Eqns. (5) and (14) and the imperfection factors $\alpha_{L T}$ and $\alpha_{i}$ $(i=y, z)$ are associated with the LT and FT buckling curves according to EN 1993-1-1 [5], respectively. It is important to note that the imperfection factor $\alpha_{y}$ or $\alpha_{z}$ corresponds to the buckling curve used for buckling about $y-y$ or $z-z$ axis and takes the value $\alpha_{i}=\alpha_{y}$ when $\min \left(\chi_{y}, \chi_{z}, \chi_{T}\right)=\chi_{y}$ and $\alpha_{i}=\alpha_{z}$ when $\min \left(\chi_{y}, \chi_{z}, \chi_{T}\right)=\chi_{z}$ or $\min \left(\chi_{y}, \chi_{z}, \chi_{T}\right)=\chi_{T}$.

The design buckling resistance and design criterion for proposed GM approach are presented in [19].

\section{Implementation of the developed GM approach}

Two I-section beam-columns are considered, namely HEB 300 and IPE 360 made of steel grade S235. Firstly, the beam columns without LT restraints are considered, next the beam-columns with LT restraints. One and seven LT restraints are used for HEB 300 section and three restraints for IPE 360. In all cases the length of elements corresponds to the number of intermediate LT restraints and relative slenderness ratio $\bar{\lambda}_{z}$ which are given at the top of the respective Figures. Two moment gradient ratios are accounted for, namely $\psi_{y}=1,0$ (uniform bending) and $\psi_{y}=-1,0$ (antisymmetric bending moment diagram). The results from the proposed GM are compared with those from Eurocode 3-1-1 interaction equations according to Method 1 and Method 2 [5] where $\chi_{L T}$ is calculated according to the General case of the clause 6.3.2.2 [5].

\subsection{Unrestrained beam-columns}

Results for the unrestrained beam-columns are given in Fig. 3 for $\psi_{y}=1,0$ and in Fig. 4 for $\psi_{y}=-1,0$. The proposed approach gives the same results as from EN 1993-1-1 [5] for two extreme load cases $m_{y, E d}=0$ and $n_{E d}=0$. For the uniform bending moment and for intermediate proportion between $m_{y, E d}$ and $n_{E d}$ the proposed resistance curves are a few percent higher than those from EN 1993-1-1 [5]. In case of antisymmetric moment diagram the results from developed GM approach and from Method 1 [5] are close to each other and both are above those obtained from Method 2 [5].

\subsection{Restrained beam-columns}

The results for HEB 300 with one intermediate LT restraint and IPE 360 with three intermediate LT restraints are given in Fig. 5 for $\psi_{y}=1,0$ and in Fig. 6 for $\psi_{y}=-1,0$.

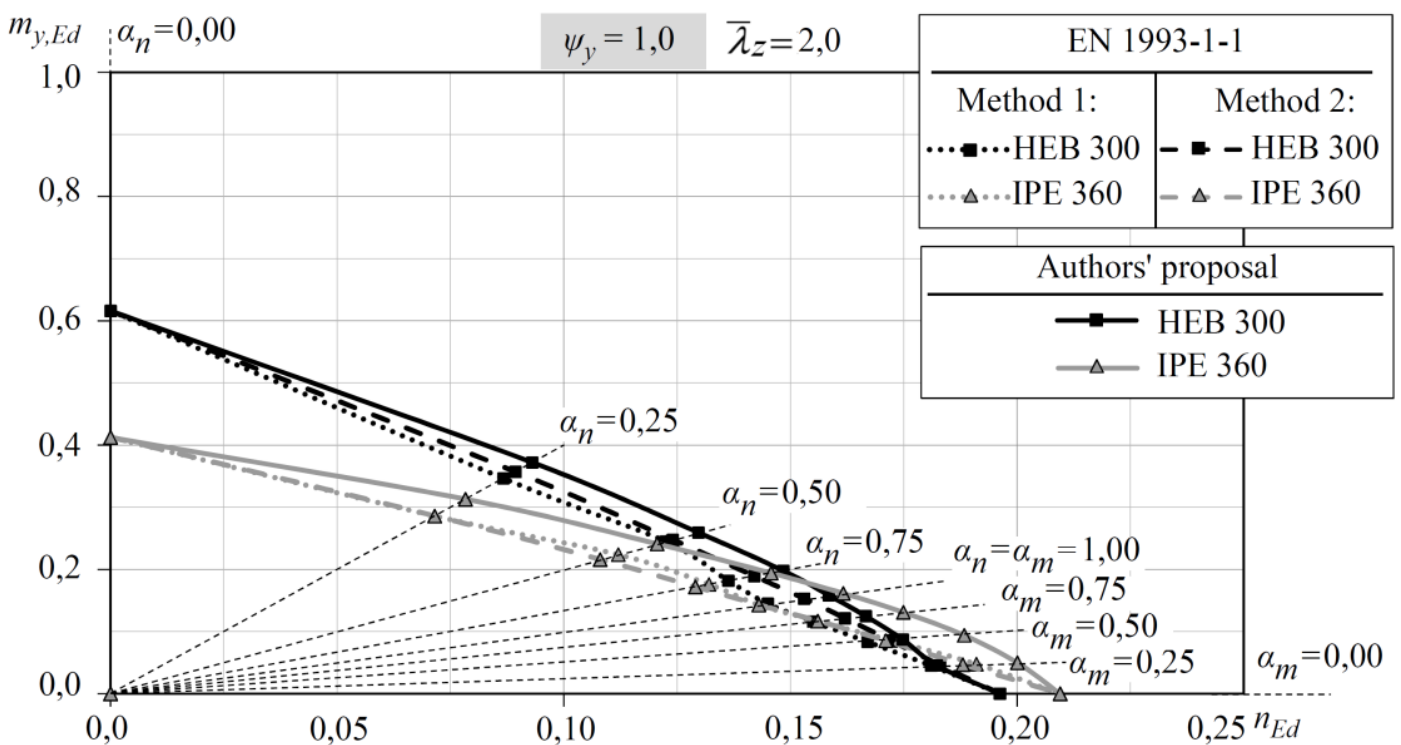

Fig. 3. Comparison of results for the unrestrained beam-columns and $\psi_{y}=1,0$. 


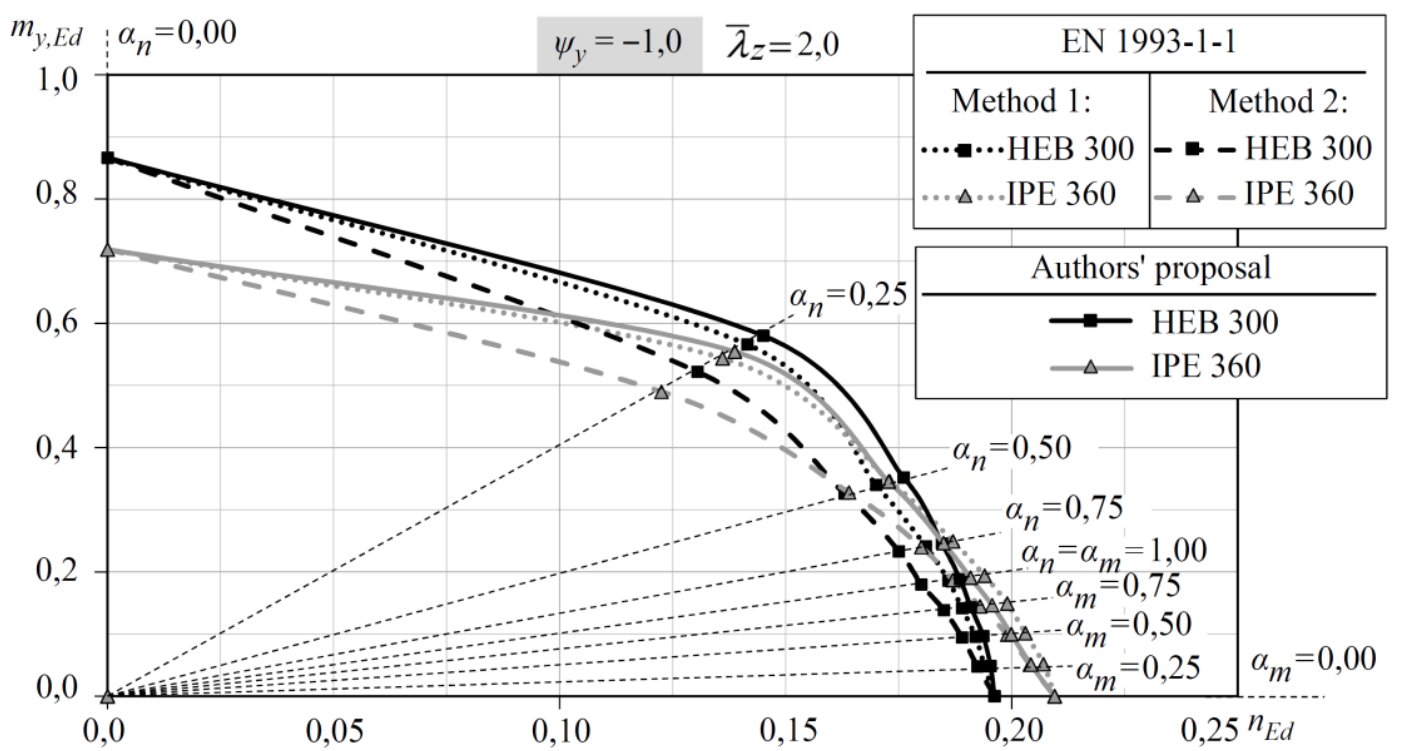

Fig. 4. Comparison of results for the unrestrained beam-columns and $\psi_{y}=-1,0$.

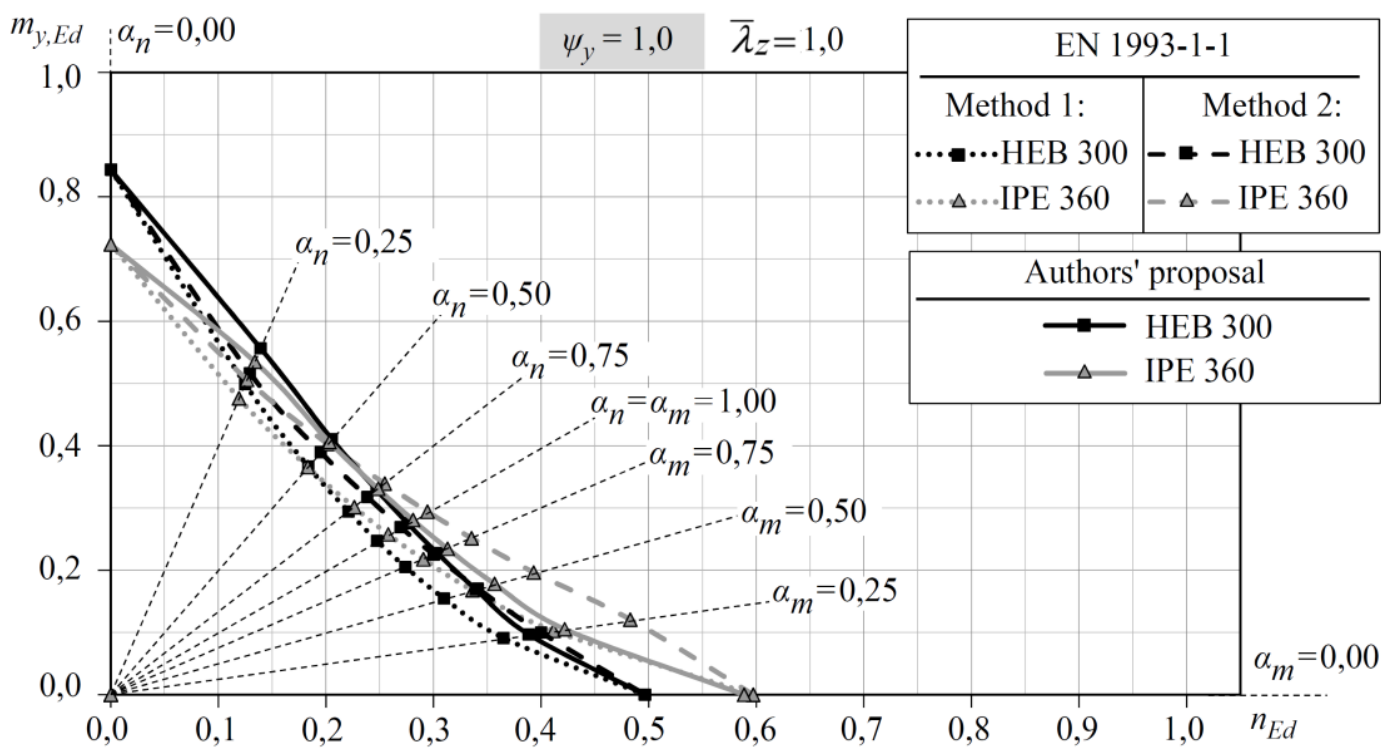

Fig. 5. Comparison of results for the beam-columns with one (HEB) or three (IPE) intermediate LT restraints and $\psi_{y}=1,0$.

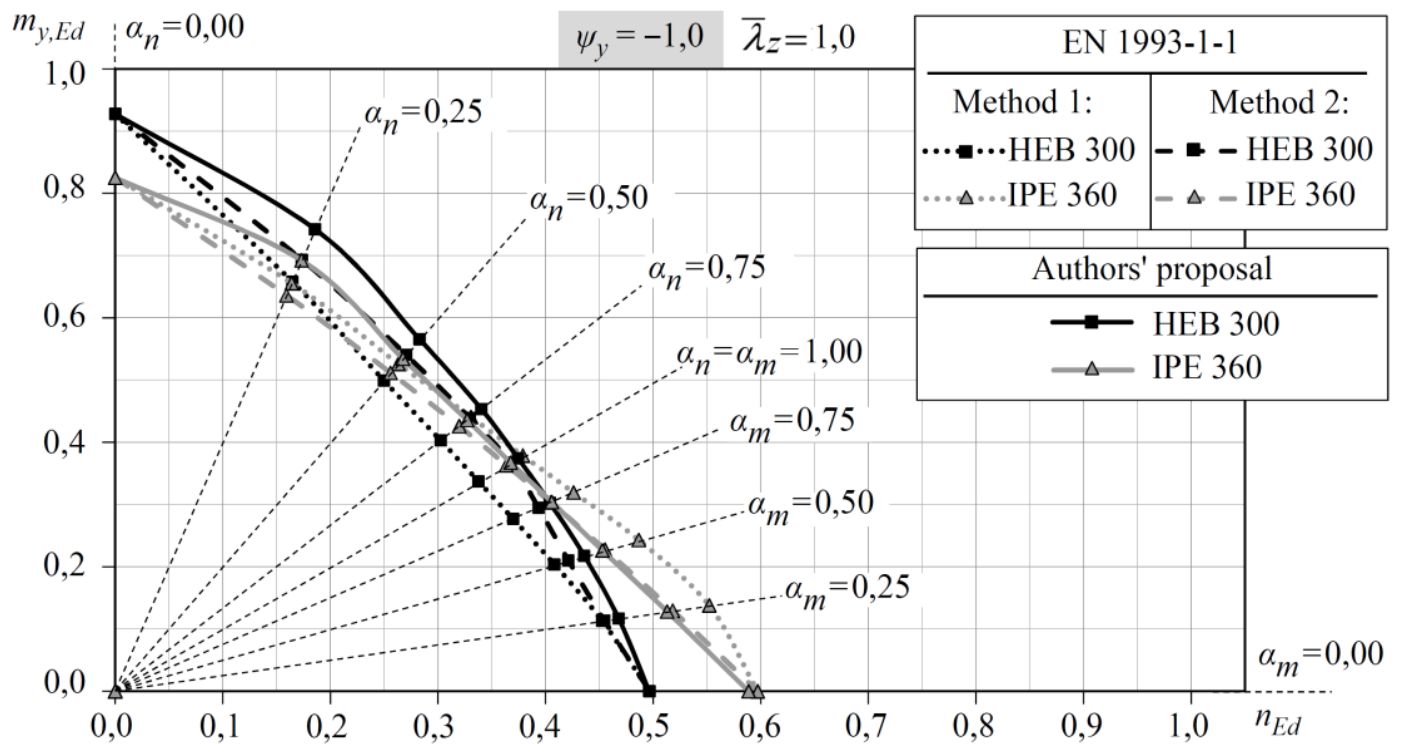

Fig. 6. Comparison of results for the beam-columns with one (HEB) or three (IPE) intermediate LT restraints and $\psi_{y}=-1,0$. 


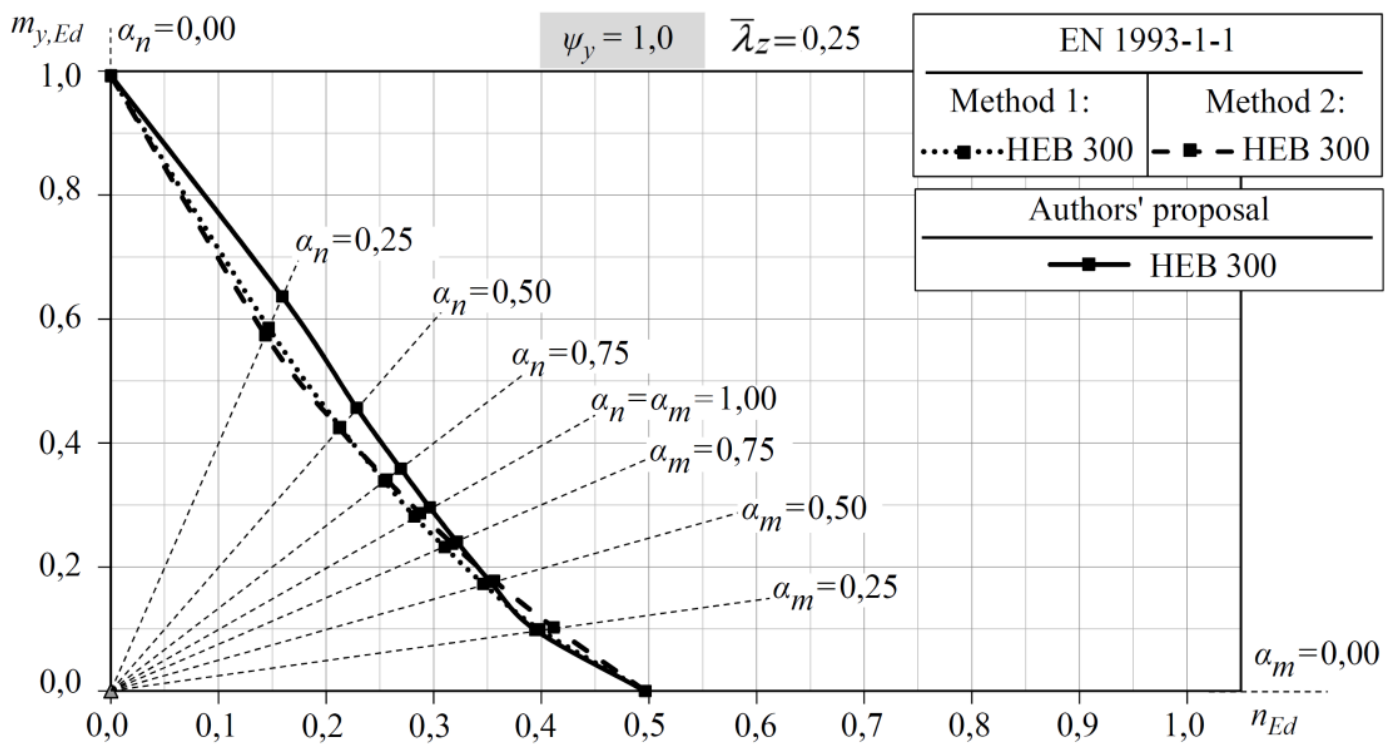

Fig.7. Comparison of results for the beam-columns with seven intermediate LT restraints and $\psi y=1,0$.

For two extreme load cases $m_{y, E d}=0$ and $n_{E d}=0$ the results from both method are the same as for the unrestrained beam-columns, except in the case of IPE 360 section and pure compression. For IPE 360 and $m_{E d}=0$ the buckling resistance from proposed approach is about 1,5\% less than from EN 1993-1-1 [5], but this small difference is only if the buckling slenderness $\bar{\lambda}_{y}$ is slightly greater than $\max \left(\bar{\lambda}_{z}, \bar{\lambda}_{T}\right)$ and the reduction factor $\chi_{y}$ is greater than $\min \left(\chi_{z}, \chi_{T}\right)$ at the same time. For intermediate proportion between $m_{y, E d}$ and $n_{E d}$ the results from the proposed GM approach are generally close or a bit higher than those from EN 1993-1-1 [5]. But for low values of $m_{y, E d}$ and IPE 360 section, the Eurocode's Method 2 in case of $\psi_{y}=1,0$ and Method 1 in case of $\psi_{y}=-1,0$ leads to results above those obtained from proposed GM approach.

The results for HEB 300 with seven intermediate LT restraint is given in Fig. 7 for $\psi_{y}=1,0$. In case of $\psi_{y}=-1,0$ the buckling resistance is controlled by the in-plane buckling resistance in the entire range of the proportion between the axial compressive force and the bending moment about $y-y$ axis. For this case of restrained beam-columns, similar conclusions can be made as for the unrestrained beam-columns.

\section{Final remarks}

This paper is a continuation of previous authors' studies [6-9] on refining the GM approach. In the paper different aspects of Eurocode 3 General Method approaches are discussed. It has been shown that GMNIA/LBA EC3-1-1 approach according to clause 6.3.4 [5] in case of axial compression is quite conservative to the interaction buckling resistance according to clause 6.3.3 [5]. The reason for the lower buckling resistance is the fact that in GMNIA/LBA EC3-1-1 approach the effect of imperfections is considered twice at their maximum magnitude, firstly the maximum effect of in-plane imperfections is considered in the calculation of $\alpha_{u l t, i p}$ and secondly, the maximum effect of out-of-plane imperfections is considered in the calculation of $\chi_{o p}$. The purpose of present study is to improve the application of GM approach for both beam-columns without intermediate LT restraints and with these restraints. In the developed GM approach, the effect of imperfections is considered only once in the calculation of $\chi_{F T, k}$ and proposed approach gives the same results as from EN 1993-1-1 [5] for two extreme load cases $m_{y, E d}=0$ and $n_{E d}=0$.

The examples of beam-columns of HEB 300 and IPE 360 sections under compression and symmetric or antisymmetric moment loading conditions are considered for unrestrained and discretely intermediate restrained cases. The results from the proposed GM approach are compared with those from Eurocode's interaction equations according to Method 1 and Method 2 [5] where $\chi_{L T}$ is calculated according to the General case of the clause 6.3.2.2 [5]. The obtained differences are discussed. The results from the proposed GM approach have generally good consistency with the results obtained from Eurocode's interaction equations [5], especially for two extreme load cases $m_{y, E d}=0$ and $n_{E d}=0$.

\section{References}

1. A. Boissonnade, J.-P. Jaspart, J.-P. Muzeau, M. Villette, New interaction formulae for beamcolumns in Eurocode 3: The French-Belgian approach, J. Constr. Steel Res., 60, 421-431 (2004)

2. R. Greiner, J. Lindner, Interaction formulae for members subjected to bending and axial compression in Eurocode 3 - the Method 2 approach, J. Constr. Steel Res., 62, 757-77 (2006)

3. F. Bijlaard, M. Feldmann, J. Naumes, G. Sedlacek, The "general method" for assessing the out-ofplane stability of structural members and frames and the comparison with alternative rules in 
EN 1993 - Eurocode 3 - Part 1-1, Steel Constr., 3, 19-33 (2010)

4. Eurocode 3. EN 1993-1-6: Design of Steel Structures, Part 1-6: Strength and stability of shell structures, CEN (2007)

5. Eurocode 3. EN 1993-1-1: Design of Steel Structures, Part 1-1: General rules and rules for buildings, CEN (2005)

6. M. A. Gizejowski, Z. Stachura, A consistent Ayrton-Perry approach for the flexural-torsional buckling resistance evaluation of steel I-section members, CEER, 2, 89-105 (2017)

7. Z. Stachura, M. A. Gizejowski, An alternative analytical-numerical procedure in Eurocode's design of steel members with discrete lateral and torsional in-span restraints [in Polish], JCEEA, 64, 471-486 (2017)

8. M. A. Gizejowski, Z. Stachura, Flexural-torsional buckling resistance of steel I-section beam-columns in context of structural Eurocodes evolution [in Polish], IiB, 6, 303-308 (2017)

9. M. A. Gizejowski, Z. Stachura, Generalized AyrtonPerry approach for the evaluation of beam-column resistance. In: Insights and Innovations in Structural Engineering, Mechanics and Computation, 253-254 (e-book on CD,713-719) (London: Taylor \& Francis Group 2016)

10. M. Nedelcu, Generalisation of the Ayrton-Perry formula for the global-distortional-local buckling of thin-walled members, Thin-Walled Struct., 118, 73-86 (2017)

11. T. Tankova, L. Marques, A. Andrade, L. Simoes da Silva, A consistent methodology for the out-of-plane buckling resistance of prismatic steel beamcolumns, J. Constr. Steel Res., 128, 839-852 (2017)

12. J. Szalai, Complete generalization of the AyrtonPerry formula for beam-column buckling problems, Eng. Struct., 153, 205-223 (2017)

13. F. Papp, Buckling assessment of steel members through overall imperfection method, Eng. Struct., 106, 124-136 (2016)

14. N. Boissonnade, M. Hayeck, E. Saloumi, J. Nseir, An overall interaction concept for an alternative approach to steel members design, J. Constr. Steel Res., 135, 1-41 (2017)

15. B.W. Schafer, Review: the direct strength method of cold-formed steel member design, J. Constr. Steel Res., 64, 766-778 (2008)

16. J. Ferreira, P. V. Real, C. Couto, Comparison of the General Method with the Overall Method for the out-of-plane stability of members with lateral restraints, Eng. Struct., 151, 153-172 (2017)

17. F. Mohri, Ch. Bouzerira M. Potier-Ferry, Lateral buckling of thin-walled beam-column elements under combined axial and bending loads, ThinWalled Struct., 46, 290-302 (2008).

18. N.S. Trahair, M.A. Bradford, D.A. Nethercot, L. Gardner, The behaviour and design of steel structures to EC3 (London - New York: Taylor \& Francis 2008)

19. Z. Stachura, M. A. Gizejowski, Partial factors in modelling of steel structures reliability according to Eurocodes, CEER, 16, 195-207 (2015) 